Eneida Márcia de Souza Simões 1 Michael E. Reichenheim 2

\section{Confiabilidade das informações de causa básica nas declarações de óbito por causas externas em menores de 18 anos no Município de Duque de Caxias, Rio de Janeiro, Brasil}

\author{
Reliability of information on the underlying \\ cause of death from external causes in children \\ and adolescents under 18 years of age \\ in the Municipality of Duque de Caxias, \\ Rio de Janeiro, Brazil
}

\footnotetext{
1 Divisão de Epidemiologia Secretaria Municipal de Saúde de Duque de Caxias. Alameda Dona Tereza 3 , Duque de Caxias, $R J$ 25235-000, Brasil. eneida@centoin.com.br 2 Departamento de Epidemiologia e Núcleo de Pesquisas das Violências, Instituto de Medicina Social, Universidade do Estado do Rio de Janeiro. Rua São Francisco Xavier 524, 7o andar, Rio de Janeiro, RJ 20559-900, Brasil. michael@ims.uerj.br
}

\begin{abstract}
This study assesses the quality of official data on mortality due to external causes. It examines the agreement in the underlying cause of death by accidents and violence in children and adolescents under 18 years of age in the Municipality of Duque de Caxias, Rio de Janeiro State. Codings based on death certificates (DC) issued by the Institute of Forensic Medicine - IFM $\left(I C D_{i f m}\right)$ and those complemented by the Municipal Secretariat of Health $-M S H\left(I C D_{m s h}\right)$ are compared to the coding directly obtained from autopsy logs and attached to police reports accessible at the IFM (ICD research $)$. Levels of agreement (kappa) between ICD $D_{\text {sh }} / I C D_{\text {ifm }}, I_{\text {Cesearch }}^{\prime}$ $I C D_{\text {ifm }}$, and $I C D_{\text {research }} / I C D_{m s h}$ were 0.33, 0.26, and 0.81, respectively, in 1995 and 0.26, 0.22, and 0.71 in 1996. When analyzed together, all three sources agreed by $45 \%$ and $37.6 \%$ in the respective years. ICD ${ }_{\text {research }}$ and $I C D_{m s h}$ agreed among themselves and disagreed with $I C D_{\text {ifm }}$ by $46.9 \%$ in 1995 and $48.2 \%$ in 1996. Results show that the IFM fails to transfer available information to the MSH via DCs. Although a satisfactory updating is subsequently carried out at the MSH, the relevance and legality of this procedure should be called into question and further discussed.
\end{abstract}

Key words Reproducibility of Results; Underlying Cause of Death; Violence; Accidents; Death Certificates

Resumo Este estudo visa avaliar a qualidade das informações oficiais relativas à mortalidade por causas externas, examinando a concordância sobre a causa básica das mortes por acidentes e violências em menores de 18 anos residentes no Município de Duque de Caxias, Rio de Janeiro. Compara-se as codificações feitas a partir das Declarações de Óbito (DOs) emitidas pelo Instituto Médico Legal - IML (CID ${ }_{\text {iml }}$ ) e as complementadas pela Secretaria Municipal de Saúde - SMS $\left(C I D_{s m s}\right)$ com as da pesquisa, obtidas diretamente a partir de informações dos laudos necroscópicos do IML e dos Registros de Ocorrência Policial (CID pesq). As concordâncias (kappa) encontradas entre $C I D_{\text {sms }} / C I D_{\text {iml }}$, CID $D_{\text {pesq }} / C I D_{\text {iml }}$ e $C I D_{\text {sms }} / C I D_{\text {pesq }}$ foram, respectivamente, de 0,33, 0,26 e 0,81 para 1995 e 0,26, 0,22 e 0,71 para 1996. As três fontes entre si concordaram em 45\% e 37,6\% nos respectivos anos. A CID pesq e a CID sms concordaram entre si e discordaram da $C I D_{i m l}$ em 46,9\% em 1995 e 48,2\% em 1996. Estes resultados mostram que o IML não transfere as informações que detém à SMS, via DO. Ainda que, subseqüentemente, ocorra uma satisfatória complementação das DOs na própria SMS, deve-se discutir a sua pertinência e legalidade.

Palavras-chave Reprodutibilidade de Resultados; Causa Básica de Morte; Violência; Acidentes; Atestados de Óbito 


\section{Introdução}

A magnitude crescente da violência na realidade brasileira, a partir da década de 80, é evidenciada através da mudança no perfil de mortalidade do país quando as denominadas causas externas passam de quarta para segunda causa de morte, permanecendo abaixo apenas das mortes por doenças cardiovasculares (Szwarcwald, 1987). Mello-Jorge \& Gotlieb (1998) analisando a mortalidade no Brasil no período de 1979 a 1996 verificaram, no fim da década de 70, uma taxa de 50 por $100 \mathrm{mil} \mathrm{habi-}$ tantes passando para 76 por 100 mil habitantes nos últimos anos do período analisado, representando um aumento de mais de $50 \%$.

As conseqüências desse fenômeno em termos de mortes prematuras, danos físicos e psíquicos, incapacidades e a repercussão na qualidade e cobertura dos serviços de saúde configuram no Brasil, a partir da década de 80 , um grave problema de saúde pública (Minayo, 1994; Souza, 1994). Em relação às mortes prematuras no Estado do Rio de Janeiro, Reichenheim \& Werneck (1994) constataram que o maior risco de perder anos de vida em potencial na população era devido principalmente à ação das causas externas, responsáveis por mais de $30 \%$ dos anos potenciais de vida perdidos (APVP) entre as idades de 1 a 70 anos.

Embora com repercussões legais, sócioeconômicas e políticas, é o setor saúde que arca com o peso das conseqüências da violência, como bem aponta a Organização Pan-Americana de Saúde (OPAS) no documento Saúde e Violência: Plano de Ação Regional, "o setor saúde constitui a encruzilhada para onde convergem todos os corolários da violência, pela pressão que exercem suas vitimas sobre os serviços de urgência, atenção especializada, reabilitação física, psicológica e de assistência social" (OPS, 1994:1). Ademais, aponta o documento que “(...) o setor saúde detém também um importante papel no registro de informações sobre os eventos violentos" (OPS, 1994:1).

Segundo a OPAS, o cenário ideal para a concretização do Plano de Ação Regional é o município, espaço de aproximação entre cidadãos e instituições governamentais e não governamentais. É o local apropriado para dirimir conflitos, reduzir as ineqüidades e direcionar propostas para o benefício coletivo. A participação do governo local também facilita a mobilização de todos os setores sociais para a negociação de metas, compromissos e responsabilidades operativas, técnicas e financeiras na redução da violência (OPS, 1994).
A preocupação com o impacto da violência e suas conseqüências em Duque de Caxias tem mobilizado também instâncias municipais em especial a Secretaria Municipal de Saúde (SMS) - na identificação dos fatores intervenientes e na criação de um plano de ação que contemple a prevenção e atenção às vítimas da violência.

A precariedade do sistema de informações sobre morbidade por violência e a disponibilidade de informações sobre mortalidade, tornam pertinente a avaliação do fenômeno violência através das estatísticas oficiais de mortalidade, embora as mesmas identifiquem somente o evento fatal.

O conhecimento do perfil municipal de mortalidade por causas externas, os tipos específicos de violência (suicídio, homicídio, acidentes) e qual deles se apresenta como principal causa específica no conjunto dos óbitos, permite aos planejadores analisar tendências e priorizar ações preventivas específicas. A análise $\mathrm{e}$ conhecimento do problema violência e suas especificidades no município gera, também, uma maior motivação e mobilização de recursos de instituições governamentais e não governamentais municipais na sua prevenção (OPS,1994).

As estatísticas oficiais de mortalidade são consolidadas a partir das informações presentes nas Declarações de Óbito (DO). Em caso de morte não natural - aquela que sobrevem em decorrência de um acidente, envenenamento ou violência - a lei, artigo 162 do Código de Processo Penal (Brasil, 1998), determina que esses atestados sejam emitidos por médicos legistas, após necrópsia no Instituto Médico Legal (IML) (Laurenti \& Mello-Jorge, 1983). Para efeito de estatística de mortalidade e planejamento de ações preventivas, a informação mais importante da DO é a causa básica da morte. A das mortes violentas foi definida pela Organização Mundial de Saúde (OMS, 1975:732) como "as circunstâncias do acidente ou violência que produziram a lesão fatal" ou seja, o tipo de acidente ou violência que ocasionou os ferimentos que levaram ao óbito.

Em termos de fluxo, a DO emitida pelo IML é encaminhada ao Cartório de Registro Civil onde, após o registro pertinente, é recolhida em geral pela SMS e especificamente em Duque de Caxias, pelo Núcleo de Dados Vitais (NDV).

No ano de 1995, teve início a codificação da causa básica pelos técnicos da SMS, embora com a supervisão da Secretaria Estadual de Saúde do Rio de Janeiro (SES-RJ) que cessa a partir de 1996. Os técnicos do referido Núcleo, acatando orientações vindas da esfera estadual, complementam os dados constantes na DO 
com as informações do livro de registro de necrópsias do IML e, somente então, codificam a causa básica da morte. Subseqüentemente, as informações são encaminhadas à SES-RJ. Esses dados, portanto, correspondem às estatísticas oficiais do Município de Duque de Caxias.

Estudos anteriores evidenciaram a deficiência qualitativa e quantitativa da informação oficial relativa às causas de morte em geral (Carvalho \& Silver, 1995; Fonseca \& Laurenti, 1974; Monteiro et al., 1997a, 1997b; Moriyama, 1989; Puffer \& Griffith, 1968), e as mortes por causas externas em particular (Ladeira \& Guimarães, 1998; Laurenti \& Mello-Jorge, 1987; Mello-Jorge, 1982, 1988, 1990; Njaine et al., 1997; Souza, 1991; Zaluar et al.,1994). Esses estudos identificaram que o evento violência não é devidamente elucidado pela precariedade da investigação policial, ou pelo preenchimento incompleto ou mesmo incorreto da DO pelo médico legista. No âmbito judicial, estes fatos podem estar contribuindo para a impunidade dos crimes cometidos. Na esfera das políticas públicas, ao distorcerem as estatísticas oficiais relativas à mortalidade por causas externas, implicam em proposição e adoção de medidas preventivas ou assistenciais inadequadas.

Como a fonte oficial de informações sobre violência são as estatísticas de mortalidade provenientes da DO, justifica-se esse estudo. A identificação da confiabilidade dessas informações, ao subscrever a fidedignidade do perfil da violência, certamente viria a fortalecer as ações de prevenção. Por outro lado, o reconhecimento de distorções nas informações da DO, encorajaria uma reclassificação dos óbitos e, mediante uma possível mudança do perfil da violência, viabilizaria a reorientação de prioridades quanto às ações de prevenção.

Nessa perspectiva, o objetivo do presente estudo é avaliar a concordância das informações sobre a causa básica da morte por acidentes e violências em menores de 18 anos residentes em Duque de Caxias, comparando as codificações feitas a partir das DO emitidas pelo IML e as DO complementadas pela Secretaria Municipal de Saúde de Duque de Caxias (SMS-DC), com informações diretamente coletadas, de forma independente, nos laudos necroscópicos do IML e nos Registros de Ocorrência Policial (ROP).

\section{Material e métodos}

A avaliação de confiabilidade baseia-se nos óbitos por causas externas de residentes em Duque de Caxias menores de 18 anos ocorridos em 1995 e 1996. O ano de 1995 foi escolhido por ter sido o último em que foi utilizada a codificação pela Classificação Internacional de Doenças (CID) - 9a Revisão possibilitando, portanto, uma comparação com dados de pesquisas anteriores (Mello-Jorge, 1990; Souza, 1991). Optou-se por 1996 por ter sido esse o ano inicial da utilização da CID-10a Revisão.

O conhecimento mais sistematizado que se tem sobre violência, encontra-se nas informações sobre mortalidade por causas externas. $\mathrm{O}$ termo causas externas refere-se, nas duas revisões da CID, aos fatores externos ao organismo humano que provocam lesões, envenenamentos ou efeitos adversos no homem. Esse grupo de causas, categorizado de acordo com a "Classificação Suplementar de Causas Externas de Lesões e de Envenenamentos" da CID-9a Revisão, compreende os códigos E800 - E999 (OMS, 1975). Na CID-10a Revisão, a denominação passa a ser "Causas Externas de Morbidade e Mortalidade", compreendendo os códigos V01 Y98 (OMS, 1993).

As mortes por causas externas de residentes em Duque de Caxias mas que tenham ocorrido fora do município não foram contempladas no presente estudo em virtude de ter-se escolhido o IML local como fonte de dados. O mesmo ocorre com os óbitos de residentes ocorridos em Duque de Caxias, mas que tenham sido registrados em cartórios de outros municípios, não sendo portanto codificados pelo NDV da SMS-DC.

O material utilizado foi constituído das informações provenientes das DO, laudos necroscópicos e do ROP, que geralmente acompanha o corpo a ser necropsiado. A descrição do material, assim como o método de trabalho utilizado, encontram-se a seguir.

\section{Coleta de informações}

- Instituto Médico Legal - Após consulta ao livro de registro das necrópsias realizadas em 1995 e 1996, foram selecionadas todas as fichas de entrada do corpo de menores de 18 anos no IML, bem como as dos corpos não identificados. Os laudos necroscópicos e os respectivos ROPs foram manuseados um a um visando avaliar sua elegibilidade. Os casos que, após a necrópsia e investigação policial auxiliada pela datiloscopia, permaneceram sem identificação foram excluídos, mesmo que tivessem a idade presumível fornecida pelo perito. Essa exclusão foi por não se ter como ratificar a residência no Município de Duque de Caxias. Também foram excluídos os óbitos rotulados pela ne- 
crópsia como decorrentes de evolução de processo mórbido ou de causa indeterminada.

No ano de 1995, registros informam que deram entrada no IML 1.771 corpos para serem necropsiados, sendo que apenas 127 não puderam ser identificados. Foram realizadas 329 necrópsias em menores de 18 anos. Desse total, descartou-se 215 laudos por atenderem aos critérios de exclusão. Ocorreram três perdas por não localização dos laudos, dois no IML e um na SMS-DC.

Do total de 1.673 corpos necropsiados no IML em 1996, 120 não foram identificados e 296 eram de menores de 18 anos. Por contemplarem os critérios de exclusão foram descartados 198 laudos. Embora constassem no livro de registro de necrópsias, ocorreram treze perdas, dez por não localização dos laudos no IML, um na SMS-DC e dois em os ambos serviços.

As informações constantes nos laudos necroscópicos e nos ROPs dos óbitos foram transcritas para uma ficha de coleta de informações especificamente elaborada para o estudo (Ficha de Coleta de Dados do IML). Essa ficha contempla dados de identificação da vítima (nome, sexo, data do nascimento, cor, endereço, data do óbito e da necrópsia), da delegacia de polícia, do registro de ocorrência policial, da circunstância e conseqüência do evento.

A partir dessas informações, a causa básica da morte foi codificada pelos técnicos do NDV da SMS-DC, os mesmos que codificaram a causa básica das DOs. A participação dos codificadores da SMS-DC visou preservar a qualidade e equivalência da codificação, permitindo que se descartasse este fator como um possível interveniente nos resultados da avaliação de confiabilidade. A codificação se deu de forma mascarada em relação às informações contidas na DO e seguiu rigorosamente a CID-9a Revisão para os laudos emitidos em 1995 e a CID-10a Revisão para os emitidos em 1996. Estas informações passam ser referidas como $\mathrm{CID}_{\text {pesq }}$ no restante do texto.

- Secretaria Municipal de Saúde - No ano de 1995 foram codificadas 119 DOs por causas externas. Oito foram excluídas pelos seguintes motivos: emissão indevida pelo hospital que atendeu à vítima sem ter sido o corpo encaminhado ao IML para necrópsia (1); causa indeterminada (5) e não localização no IML (2). Na análise foram efetivamente utilizadas 111 codificações. Em 1996 foram codificadas 98 DOs por causas externas. Treze foram excluídas, dez por não localização no IML e três por causa indeterminada. A análise contemplou efetivamente 85 codificações.

As informações referentes à identificação da vítima, causa da morte e tipo de violência (homicídio, suicídio, acidente ou ignorado) contidas na DO e preenchida pelo legista foram transcritas para uma outra ficha de coleta de informações (Ficha de Coleta de Dados da DO). Posteriormente solicitou-se também aos técnicos que atuam no NDV da SMS-DC que codificassem a causa básica extraída desse formulário. Esses dados passam doravante a ser denominados $\mathrm{CID}_{\text {iml }}$.

Conforme indicado anteriormente, a DO emitida pelo IML é complementada pelos codificadores do NDV da SMS-DC com informações obtidas do livro de registro de necrópsias do IML. Com estas informações adicionais, procede-se a codificação da causa básica da morte, classificação que passa a corresponder às estatísticas oficiais de Duque de Caxias. O código dado à causa básica da DO complementada foi copiado para uma terceira ficha de coleta de informações (Ficha de Coleta de Dados da DO Complementada). Estes dados passam a ser referidos como $\mathrm{CID}_{s m s}$.

\section{Qualidade da informação}

O controle da qualidade do processo de coleta de dados no IML foi avaliado numa fase anterior ao trabalho de campo e no início e meio da de coleta de dados. As informações constantes nos laudos necroscópicos e nos ROPs de 35 óbitos - 15 em 1994, 10 em 1995 e 10 em 1996 foram transcritas para a Ficha de Coleta de Dados do IML. Com base nessas informações, a autora principal (EMSS) e uma colaboradora previamente treinada, de forma independente e mascarada, consolidaram a causa básica e a registraram na própria Ficha.

A concordância obtida em relação à causa básica, analisada por um consultor externo, foi considerada substancial segundo a qualificação de Shrout (detalhes adiante) - kappa =0,96 (IC 95\%: 0,80 - 1,00). Essa avaliação indicou uma adequada padronização do processo de coleta de dados, permitindo dar prosseguimento ao trabalho de campo realizado exclusivamente pela primeira autora (EMSS).

\section{Processamento e análise dos dados}

Após a entrada de dados, realizou-se uma controle sistemático da qualidade da digitação mediante entrada dupla e checagem eletrônica (Epi Info- CDC, 1990). Em caso de diferença eram consultadas as fichas para a correção. A análise contemplou o contraste em nível de 30 dígito ou categoria, e de agrupamento da CID. 
Visando estabelecer em que medida as três fontes pesquisadas (SMS, IML e Pesquisa) apresentavam as mesmas informações quanto a causa básica da morte, foram realizadas análises de freqüência para avaliar a concomitância de identificação pelas três fontes. Nas avaliações formais de concordância através de análises bivariadas utilizou-se o kappa como estimador (Cohen, 1960). Este é considerado adequado quando se tratam de variáveis categóricas avaliadas por julgadores operando independentemente. Os cálculos dos intervalos de confiança do kappa baseiam-se no método proposto por Fleiss et al. (1969).

A interpretação do kappa segue a orientação de Shrout (1998) que, à guisa de uma crítica e revisão dos adjetivos originalmente propostos por Landis \& Koch (1977), propõe a seguinte classificação: $\kappa<0,1 \rightarrow$ concordância (virtualmente) ausente; $\kappa=0,10$ a $0,40 \rightarrow$ concordância fraca $($ slight $) ; \kappa=0,41$ a $0,60 \rightarrow$ concordância discreta (fair); $\kappa=0,61$ a $0,80 \rightarrow$ concordância moderada; $\kappa=0,81$ a $1,0 \rightarrow$ concordância substancial.

\section{Resultados}

Nas Tabelas 1 e 3 encontram-se, respectivamente, as categorias das CID-9 e CID-10 contempladas na análise. Também são indicadas as correspondentes abreviaturas usadas nas Tabelas 2 e 4 . Nestas tabelas (1o e 2o números de cada casela; ver detalhes nas notas de rodapé das tabelas), encontram-se os perfis de concordância quanto à classificação da causa básica entre o IML e as duas outras fontes (SMS e Pesquisa), respectivamente para os anos de 1995 e 1996. Em 1995, os estimadores de ponto dos kappas estão em torno de 0,3 , e na melhor das circunstâncias, não ultrapassam 0,4 ao se considerar os limites superiores de confiança. Em 1996, a concordância é ainda menor com estimativas caindo em cerca de 10 a 15\%. Nesse ano, as estimações de ponto não ultrapassam 0,26.

Quando se compara as classificações da SMS e da Pesquisa (3o número de cada casela nas Tabelas 2 e 4; ver detalhes nas notas de rodapé das tabelas) pode-se observar um aumento substantivo. Os valores de kappa sobem para 0,81 em 1995 (Tabela 2) e 0,71 em 1996 (Tabela 4).

Deve ser notado que as principais discordâncias encontradas entre o IML e a SMS ou a Pesquisa referem-se aos homicídios e acidentes de trânsito. Em 1995, a SMS e a Pesquisa classificaram, respectivamente, 45 e 46 óbitos como sendo homicídio por arma de fogo, o que discorda do IML que os classificou como acidente por arma de fogo (7); ataque por procedimentos especificados e não especificados (16) e lesão por outros meios e os não especificados da qual se ignora se foi acidental ou intencionalmente infligida (22 entre a SMS e o IML e 23 entre a Pesquisa e o IML).

Em 1996, a SMS e a Pesquisa classificaram 32 óbitos como sendo homicídio por arma de fogo, novamente divergindo do IML que os classificou como agressão por meio de objeto contundente (8); agressão por meio de objeto cortante ou penetrante (um entre a Pesquisa e o IML); disparo de arma de fogo com intenção não determinada (seis entre a SMS e o IML e sete entre a Pesquisa e o IML); e fatos ou eventos não especificados com intenção não determinada (18 entre a SMS e o IML e 16 entre a Pesquisa e o IML).

Em relação aos acidentes de trânsito ocorridos em 1995, a SMS e a Pesquisa identificaram um óbito por colisão entre veículos a motor, classificado pelo IML como acidente por outras causas; e cinco óbitos por atropelamento, dos quais quatro o IML classificou como acidentes por outras causas e um como lesão por outros meios do qual se ignora se acidental ou intencionalmente infligidas. O mesmo ocorreu em 1996 quando a SMS e a Pesquisa identificaram nove atropelamentos que não foram classificados pelo IML como tal.

As Tabelas 5 e 6 mostram, respectivamente para os anos de 1995 e 1996, os resultados obtidos quando se comparam as causas básicas das três fontes estudadas. Em nível de CID terceiro dígito, o percentual de concordância completa cai de $45 \%$ em 1995 para 37,6\% em 1996. Em relação ao agrupamento da CID, a queda anual foi de $59,5 \%$ para $52,9 \%$.

Em nível de CID terceiro dígito (Tabelas 5 e 6), a codificação da Pesquisa e da SMS foi idêntica em 91,9\% das causas básicas em 1995, diminuindo ligeiramente em 1996 (85,8\%). À classificação por agrupamento de CID, no entanto, as duas fontes concordaram de forma consistente nos dois anos estudados (c. 92,8\%).

\section{Discussão}

A fraca concordância entre o IML, tanto em relação à SMS quanto à Pesquisa, corroboram a hipótese inicial de que as informações enviadas à instituição responsável pelas estatísticas oficiais são inadequadas. Por vezes, somente é descrito o fato violência, sem maiores detalhes sobre a modalidade ou meio pelo qual foi co- 
Tabela 1

Relação das causas básicas por causas externas segundo a CID-9ạ Revisão (incluindo abreviaturas utilizadas na Tabela 2).

\begin{tabular}{|c|c|c|}
\hline Causa Básica & CID & Abreviaturas* \\
\hline Acidente por queda dentro de, sobre, ou de um trem & E804 & a.trem \\
\hline Outros acidentes de trânsito por colisão entre veículo a motor e outro veículo a motor & E812 & a.veic \\
\hline Acidente de trânsito por colisão entre veículo a motor e um pedestre & E814 & a.ped \\
\hline Afogamento e submersão acidentais & E910 & a.afog \\
\hline Acidentes causados por arma de fogo & E922 & a.arma \\
\hline Acidente causado pela corrente elétrica & E925 & a.eletr \\
\hline Outros acidentes por outras causas, e as não especificadas, ambientais e naturais & E928 & a.oc \\
\hline Suicídio e lesão auto-infligida por enforcamento, estrangulamento e sufocação & E953 & suicid1 \\
\hline Suicídio e lesão auto-infligida por outros procedimentos e pelos não especificados & E958 & suicid2 \\
\hline Homicídio por estrangulamento e enforcamento & E963 & agr.est \\
\hline Agressão com armas de fogo ou explosivos & E965 & agr.arm \\
\hline Agressão com instrumentos cortantes e penetrantes & E966 & agr.faca \\
\hline Agressão por outros procedimentos especificados e pelos não especificados & E968 & agr.oc \\
\hline Lesão por submersão (afogamento) da qual se ignora se foi acidental ou intencionalmente infligida & E984 & ind.afog \\
\hline Lesão por armas de fogo e explosivos, da qual se ignora se foi acidental ou intencionalmente infligida & E985 & ind.arm \\
\hline Lesão por outros meios e os não especificados, da qual se ignora se foi acidental ou intencionalmente infligida & E988 & ind.om \\
\hline
\end{tabular}

* Abreviaturas usadas na Tabela 2.

Tabela 2

Concordância da causa básica, de acordo com a CID (terceiro dígito), entre Secretaria Municipal de Saúde,

Pesquisa e Instituto Médico Legal dos óbitos por causas externas em menores de 18 anos residentes em Duque de Caxias, 1995.

\begin{tabular}{|c|c|c|c|c|c|c|c|c|c|c|c|c|c|c|c|c|c|c|}
\hline $\begin{array}{l}\text { Causa } \\
\text { Básica* }\end{array}$ & a.trem & a.veic & a.ped & a.afog & a. arma & a.eletr & a.oc & suicid1 & suicid2 & agr.est & & gr.arn & & agr.faca & agr.oc & ind.afog & ind.arm & ind.om \\
\hline a.trem & 000 & & & & & & & & & & & & & & & & & \\
\hline a.veic & & 001 & & & & & & & & & & & & & & & & \\
\hline a.ped & & & 005 & & & & & & & & & & & & & & & \\
\hline a.afog & & & & 967 & & & & & & & & & & & & 030 & & \\
\hline a.arma & & & & & 000 & & & & & & 7 & 7 & 0 & & & & & \\
\hline a.eletr & 101 & & & & & 343 & & & & & & & & & & & & \\
\hline a.oc & & 110 & 440 & & & & 000 & & & & & & & & & & & \\
\hline suicid1 & & & & & & & & 100 & & & & & & & & & & 010 \\
\hline suicid2 & & & & & & & & & 111 & & & & & & & & & \\
\hline agr.est & & & & & & & & & & 001 & & & & & & & & \\
\hline agr.arm & & & & & & & & & & & 37 & 37 & 81 & & & & 001 & 001 \\
\hline agr.faca & & & & & & & & & & & 0 & 0 & 1 & 222 & & & & \\
\hline agr.oc & & & & & & & & & & 110 & 16 & 16 & 0 & & 100 & & & 010 \\
\hline ind.afog & & & & 113 & & & & & & & & & & & & 000 & & \\
\hline ind.arm & & & & & & & & & & & 0 & 0 & 0 & & & & 000 & \\
\hline ind.om & & & 110 & & & & & 001 & & & 22 & 23 & 0 & 010 & 001 & & 100 & 211 \\
\hline
\end{tabular}

* Identificação das abreviaturas da causa básica e respectivo CID encontram-se na Tabela 1.

Nota: os três números em cada célula correspondem à confluência de diagnósticos de causa básica (CID terceiro dígito) entre, respectivamente:

- SMS (coluna) e IML (linha) $\left[\mathrm{k}_{\text {sms } / \text { iml }}=0,33\right.$ (IC 95\%: 0,27 - 0,39)]

- Pesquisa (coluna) e IML (linha) [ $k_{\text {pesq/iml }}=0,26$ (IC 95\%: 0,20-0,32)]

- SMS (coluna) e Pesquisa (linha) $\left[k_{\text {sms } / \text { pesq }}=0,81\right.$ (IC 95\%: 0,71-0,91)] 
Relação das causas básicas por causas externas segundo a CID-10ạ Revisão (incluindo abreviaturas utilizadas na Tabela 4).

\begin{tabular}{|c|c|c|}
\hline Causa Básica & CID & Abreviaturas* \\
\hline Outras causas mal definidas e as não especificadas de mortalidade & R99 & c.desc \\
\hline Pedestre traumatizado em outros acidentes de transporte e em acidentes de transporte não especificados & V09 & a.ped \\
\hline Queda acidental sem especificação & W19 & a.qda \\
\hline Exposição a outras forças mecânicas inanimadas e as não especificadas & W49 & a.fmi \\
\hline Afogamento e submersão acidentais não especificados & W74 & a.afog \\
\hline Exposição a corrente elétrica não especificada & W87 & a.eletr \\
\hline Lesão autoprovocada intencionalmente por disparo de arma de fogo de mão & $\mathrm{X} 72$ & suicid1 \\
\hline Lesão autoprovocada intencionalmente por disparo de outra arma de fogo e de arma de fogo não especificada & $\mathrm{X} 74$ & suicid2 \\
\hline Agressão por meio de enforcamento, estrangulamento e sufocação & X91 & agr.est \\
\hline Agressão por meio de disparo de outra arma de fogo ou de arma de fogo não especificada & X95 & agr.arm \\
\hline Agressão por meio de objeto cortante ou penetrante & X99 & agr.faca \\
\hline Agressão por meio de objeto contundente & Y00 & agr.con \\
\hline \multicolumn{3}{|l|}{ Envenenamento (intoxicação) por exposição a narcóticos e a psicodislépticos (alucinógenos), } \\
\hline não classificados em outra parte, intenção não determinada & $\mathrm{Y} 12$ & ind.nar \\
\hline Enforcamento, estrangulamento e sufocação, intenção não determinada & Y20 & ind.est \\
\hline Disparo de outra arma de fogo e de arma de fogo não especificada, intenção não determinada & Y24 & ind.arm \\
\hline Contato com objeto cortante ou penetrante, intenção não determinada & Y28 & ind.faca \\
\hline Contato com objeto contundente, intenção não determinada & Y29 & ind.con \\
\hline Fatos ou eventos não especificados, intenção não determinada & Y34 & ind.ne \\
\hline
\end{tabular}

* Abreviaturas usadas na Tabela 4.

Tabela 4

Concordância da causa básica, de acordo com a CID (terceiro dígito), entre Secretaria Municipal de Saúde, Pesquisa e Instituto Médico Legal dos óbitos por causas externas em menores de 18 anos residentes em Duque de Caxias, 1996.

\begin{tabular}{|c|c|c|c|c|c|c|c|c|c|c|c|c|c|c|c|c|c|c|}
\hline $\begin{array}{l}\text { Causa } \\
\text { Básica* }\end{array}$ & c.desc & a.ped & a.qda & a.fmi & a.afog & a.eletr & suicid1 & suicid2 & $\begin{array}{l}\text { agr. } \\
\text { est }\end{array}$ & & $\begin{array}{l}\text { agr. } \\
\text { arm }\end{array}$ & $\begin{array}{l}\text { agr. } \\
\text { faca }\end{array}$ & $\begin{array}{l}\text { agr. } \\
\text { con }\end{array}$ & $\begin{array}{l}\text { ind. } \\
\text { nar }\end{array}$ & $\begin{array}{l}\text { ind. } \\
\text { est }\end{array}$ & $\begin{array}{l}\text { ind. } \\
\text { arm }\end{array}$ & $\begin{array}{l}\text { ind. } \\
\text { fac }\end{array}$ & $\begin{array}{l}\text { ind. } \\
\text { con }\end{array}$ \\
\hline c.desc & 100 & & & & & & & & & & & & & 010 & & & & \\
\hline a.ped & & 009 & & & & & & & & & & & & & & & & \\
\hline a.qda & & & 001 & & & & & & & & & & & & & & & \\
\hline a.fmi & & 330 & 110 & 000 & & & & & & 0 & 01 & & & & & & & \\
\hline a.afog & & & & & 111 & & & & & & & & & & & & & \\
\hline a.eletr & & & & & & 111 & & & & & & & & & & & & \\
\hline suicid1 & & & & & & & 000 & & & & & & & & & & & \\
\hline suicid2 & & & & & & & 001 & 000 & & & & & & & & & & \\
\hline agr.est & & & & & & & & & 100 & & & & & & 010 & & & \\
\hline agr.arm & & & & & & & & & & 29 & 2858 & 001 & & & & 001 & & \\
\hline \multicolumn{19}{|l|}{010} \\
\hline agr.faca & & & & & & & & & & 0 & 10 & 222 & 101 & & & & & \\
\hline ind.nar & 001 & & & & & & & & & & & & & 000 & & & & \\
\hline ind.est & & & & & & & & & 001 & & & & & & 000 & & & \\
\hline ind.arm & & & & & & & 100 & 010 & & 6 & 70 & & & & & 200 & & \\
\hline \multicolumn{19}{|l|}{010} \\
\hline ind.fac & & & & & & & & & & & & 110 & & & & & 000 & \\
\hline ind.con & & & & & & & & & & & & & & & & & & 000 \\
\hline ind.ne & 000 & 660 & & 010 & & & & & & 18 & 162 & & & & & 001 & 000 & 202 \\
\hline 141 & & & & & & & & & & & & & & & & & & \\
\hline
\end{tabular}

* Identificação das abreviaturas da causa básica e respectivo CID encontram-se na Tabela 4.

Nota: os três números em cada célula correspondem à confluência de diagnósticos de causa básica (CID terceiro dígito) entre, respectivamente:

- SMS (coluna) e IML (linha) $\left[\mathrm{k}_{\text {sms/iml }}=0,25\right.$ (IC 95\%: 0,18 - 0,31)] • Pesquisa (coluna) e IML (linha) $\left[\mathrm{k}_{\text {pesq/iml }}=0,22\right.$ (IC 95\%: 0,14 - 0,29)]

- SMS (coluna) e Pesquisa (linha) $\left[k_{\text {sms }} /\right.$ pesq $=0,73$ (IC 95\%: 0,61-0,85)] Cad. Saúde Pública, Rio de Janeiro, $17(3): 521-531$, mai-jun, 2001 


\section{Tabela 5}

Concordância entre as fontes: CID Pesquisa ( $\left.C_{\text {pesq }}\right), C I D$ da Secretaria Municipal de Saúde $\left(\mathrm{CID}_{\mathrm{sms}}\right)$ e $\mathrm{CID}$ do Instituto Médico Legal $\left(\mathrm{CID}_{\mathrm{iml}}\right)$ quanto à causa básica da morte, segundo nível de comparação, dos óbitos por causas externas em menores de 18 anos residentes no Município de Duque de Caxias, 1995.

\begin{tabular}{|c|c|c|}
\hline \multirow[t]{2}{*}{ Nível de comparação/fonte (CID-9ạ) } & \multicolumn{2}{|c|}{ Freqüência } \\
\hline & $\mathrm{n}$ & $\%$ \\
\hline \multicolumn{3}{|l|}{ Terceiro Dígito } \\
\hline$C I D_{\text {pesq }}=C I D_{s m s}=C I D_{i m l}$ & 50 & 45,0 \\
\hline $\mathrm{CID}_{\text {pesq }}=\mathrm{CID}_{\text {sms }} \neq \mathrm{CID}_{i m /}$ & 52 & 46,9 \\
\hline $\mathrm{CID}_{\text {pesq }}=\mathrm{CID}_{\text {iml }} \neq \mathrm{CID}_{\text {sms }}$ & 1 & 0,9 \\
\hline $\mathrm{CID}_{\text {sms }}=\mathrm{CID}_{\text {iml }} \neq \mathrm{CID}$ pesq & 6 & 5,4 \\
\hline Todas diferentes & 2 & 1,8 \\
\hline Total & 111 & 100,0 \\
\hline \multicolumn{3}{|l|}{ Agrupamento } \\
\hline $\mathrm{CID}_{\text {pesq }}=\mathrm{CID}_{\text {sms }}=\mathrm{CID}_{i m l}$ & 66 & 59,5 \\
\hline $\mathrm{CID}_{\text {pesq }}=\mathrm{CID}_{\text {sms }} \neq \mathrm{CID}_{i m l}$ & 37 & 33,3 \\
\hline $\mathrm{CID}_{\text {pesq }}=\mathrm{CID}_{\text {iml }} \neq \mathrm{CID}_{\text {sms }}$ & 1 & 0,9 \\
\hline $\mathrm{CID}_{\text {sms }}=\mathrm{CID}_{\text {iml }} \neq \mathrm{CID}$ pesq & 7 & 6,3 \\
\hline Todas diferentes & - & \\
\hline Total & 111 & 100,0 \\
\hline
\end{tabular}

Tabela 6

Concordância entre as fontes: CID Pesquisa ( CID $\left._{\text {pesq }}\right), C I D$ da Secretaria Municipal de Saúde $\left(\mathrm{CID}_{\mathrm{sms}}\right)$ e $\mathrm{CID}$ do Instituto Médico Legal $\left(\mathrm{CID}_{\mathrm{im}}\right)$ quanto à causa básica da morte, segundo nível de comparação, dos óbitos por causas externas em menores de 18 anos residentes no Município de Duque de Caxias, 1996.

\begin{tabular}{|c|c|c|}
\hline \multirow[t]{2}{*}{ Nível de comparação/fonte (CID-10ạ) } & \multicolumn{2}{|c|}{ Freqüência } \\
\hline & $\mathrm{n}$ & $\%$ \\
\hline \multicolumn{3}{|l|}{ Terceiro Dígito } \\
\hline $\mathrm{CID}_{\text {pesq }}=\mathrm{CID}_{\text {sms }}=\mathrm{CID}_{i m \mid}$ & 32 & 37,6 \\
\hline$C I D_{\text {pesq }}=\mathrm{CID}_{\text {sms }} \neq \mathrm{CID}_{i m l}$ & 41 & 48,2 \\
\hline $\mathrm{CID}_{\text {pesq }}=\mathrm{CID}_{i m l} \neq \mathrm{CID}_{\text {sms }}$ & 4 & 4,7 \\
\hline $\mathrm{CID}_{\text {sms }}=\mathrm{CID}_{\text {iml }} \neq \mathrm{CID}_{\text {pesq }}$ & 6 & 7,1 \\
\hline Todas diferentes & 2 & 2,4 \\
\hline Total & 85 & 100,0 \\
\hline \multicolumn{3}{|l|}{ Agrupamento } \\
\hline $\mathrm{CID}_{\text {pesq }}=\mathrm{CID}_{\text {sms }}=\mathrm{CID}_{i m l}$ & 45 & 52,9 \\
\hline$C I D_{\text {pesq }}=\mathrm{CID}_{\text {sms }} \neq C \mathrm{CID}_{\text {iml }}$ & 35 & 41,2 \\
\hline $\mathrm{CID}_{\text {pesq }}=\mathrm{CID}_{i m l} \neq \mathrm{CID}_{\text {sms }}$ & 1 & 1,2 \\
\hline $\mathrm{CID}_{\text {sms }}=\mathrm{CID}_{i m \mid} \neq \mathrm{CID}$ pesq & 3 & 3,5 \\
\hline Todas diferentes & 1 & 1,2 \\
\hline Total & 85 & 100,0 \\
\hline
\end{tabular}

metido. Isto pode acarretar a codificação incorreta da causa básica. Por exemplo, se esta codificação tivesse sido baseada nas informações contidas na DO preenchida pelo IML, nenhuma vítima de acidente de trânsito teria sido identificada nos anos de 1995 e 1996, ainda que tenham ocorrido, respectivamente, seis e nove óbitos por essa causa. O mesmo ocorreria em relação aos homicídios por arma de fogo identificados pela Pesquisa (46 em 1995 e 32 em 1996).

Por outro lado, a concordância substancial (1995) ou moderada tendendo a substancial (1996) entre a SMS e a Pesquisa apontam para uma confiabilidade das informações encontradas após a complementação, pelos codificadores, dos dados da DO com as informações provenientes do livro de registro de necrópsias do IML. Ainda assim, deve ser notado que houve uma redução da concordância nos dois anos analisados que pode ser justificada pela expansão das categorias da CID-9a para a CID-10a Revisão. Trata-se de um achado aparentemente paradoxal, já que o aumento do número de categorias na CID-10a intentava melhorar a qualificação das mortes por causas externas, devido ao maior detalhamento na caracterização das circunstâncias e vítimas do evento (Laurenti, 1997). No entanto, este refinamento teve efeito contrário e parece ter piorado a qualidade das informações, uma vez que o preenchimento da DO pelo legista não contemplou as nuanças necessárias para uma classificação adequada. Os achados são plenamente compatíveis com essa hipótese. À utilização da CID10a, os codificadores na SMS passaram a ter menos informações para executar o processo de forma satisfatória, ao contrário da Pesquisa, na qual se teve acesso direto aos detalhes. Daí, a piora da concordância entre a SMS e a Pesquisa de um ano para o outro.

Os resultados do presente estudo de que o IML detém as informações necessárias para esclarecimento da causa básica da morte, mas não as repassa aos órgãos responsáveis pelas estatísticas oficiais, via DO, são bastante consistentes com achados de outros autores. Souza (1991), avaliando os óbitos por causas externas ocorridos e registrados em Duque de Caxias no ano de 1987, constatou uma concordância fraca (kappa $=0,23$ ) entre a classificação da causa básica da DO realizada pela SESRJ e a da própria pesquisa que utilizou como fonte de dados os ROPs. O que foi classificado naquela ocasião pela SES-RJ como causa básica da DO corresponde, na pesquisa atual, à classificação da SMS-DC. O encontro dos kappas 0,81 em 1995 e 0,71 em 1996 mostram uma 
melhora considerável da qualidade da informação quando os codificadores passam a complementar as DOs com os dados provenientes do IML.

Pesquisa afim realizada por Mello-Jorge (1990) sobre óbitos por acidentes e violências em menores de 15 anos residentes no município de São Paulo, ocorridos em 1985, compara a codificação dada à causa básica obtida através das informações constantes no IML - o que equivale ao $\mathrm{CID}_{\text {pesq }}$ no presente estudo - com a codificação da Fundação Sistema Estadual de Análise de Dados (SEADE), órgão responsável pelas estatísticas oficiais de São Paulo - o que corresponde ao $\mathrm{CID}_{s m s}$ no presente estudo. A autora detectou uma concordância, entre as duas fontes, de $39,3 \%$ para categorias e $74,8 \%$ para agrupamentos de CID. A baixa concordância em relação às categorias é justificada pela falta de detalhamento da informação permitindo, muitas vezes, uma codificação da causa básica somente nas categorias residuais. A concordância melhora quando são analisados os agrupamentos que abrangem as categorias com os eventos específicos e os de natureza não especificada. Esse fato pode ocorrer, por exemplo, quando o legista preenche a DO com dados que permitem codificar a causa básica como sendo acidente de trânsito de veículo a motor, de natureza não especificada (CID-9a E819) quando, na realidade, foi acidente de trânsito por colisão de veículo a motor e um pedestre (CID-9a - E814). Ambas as categorias estão incluídas no agrupamento acidentes de trânsito de veículos a motor (CID-9a - E810E819). No presente estudo, a concordância encontrada entre $\mathrm{CID}_{\text {pesq }}$ e CID $\mathrm{D}_{\text {sms }}$ de $91,9 \%$ e $85,8 \%$ para categorias e $92,8 \%$ e $94,1 \%$ para agrupamentos de CID, respectivamente para os anos de 1995 e 1996 (Tabelas 5 e 6), evidenciam melhora considerável da qualidade das informações oficiais quando as DOs passam a ser complementadas antes da codificação. Vale lembrar que, na ocasião em que foi realizada a pesquisa de Mello-Jorge, o SEADE codificava a causa básica com as informações provenientes da DO preenchida pelo legista, sem complementá-la. A comparação entre os dois estudos mais uma vez subscreve a pertinência da complementação e corrobora a hipótese de que o IML, apesar de deter informações detalhadas sobre a causa básica da morte, não as repassa na DO.

A coleta de informações sobre mortalidade por violência e também as implicações legais, policiais e médicas, tornam necessária a guarda adequada de todas as provas documentais, além de envidar esforços para o esclarecimento das circunstâncias do evento. Durante a per- manência da primeira autora (EMSS) no IML, foi possível observar as condições precárias de trabalho dos poucos profissionais do serviço e, conseqüentemente, as dificuldades operacionais quanto ao arquivamento dos laudos. Esse fato justifica a não localização dos laudos incorretamente arquivados, o que tende a excluir a hipótese de ocultação de laudos comprometedores para a polícia ou legista. Vale ressaltar que as DOs não localizadas na SMS foram procuradas também na SES e, da mesma forma, não localizadas. Não se encontrou justificativa plausível para esse ocorrido.

Para o esclarecimento da causa básica da morte é fundamental a necrópsia, os exames toxicológicos e anátomo-patológicos, além do desempenho da investigação policial. Ao manuseio dos laudos necroscópicos do IML, chama atenção o constante alerta por parte dos técnicos, quanto às precárias condições para a realização de todos os exames toxicológicos. Isto é bem captado na observação "exame toxicológico negativo, considerando as condições de trabalho disponíveis no laboratório" que, repetidamente, se encontra anexada aos laudos. Quanto aos exames anátomo-patológicos, não foram encontrados registros de sua realização nos laudos examinados. Ambas as deficiências podem contribuir para laudos necroscópicos inconclusivos, por mais que a necrópsia em si não tenha problemas.

A investigação policial da morte por causa violenta tem início, após o registro na delegacia policial, quando um perito é designado para ir ao local do incidente apurar o fato. As evidências apuradas são anotadas no ROP e uma cópia encaminhada ao IML, juntamente com o corpo da vítima e outros objetos que possam ajudar a esclarecer a causa da morte. Geralmente, o tempo decorrido entre a investigação policial preliminar e a necrópsia é de, no máximo, 24 horas e pretende esclarecer o tipo de acidente ou violência que ocasionou os ferimentos além de fornecer subsídios para o médico legista definir a causa básica da morte. Como as informações do ROP podem ajudar o médico legista no esclarecimento da causa básica da morte, é fundamental elucidar, em estudos futuros, a qualificação dos peritos e as condições técnicas existentes para a realização da investigação policial, em tempo hábil. Outra dificuldade ao desempenho da investigação policial é a "lei do silêncio", onde a comunidade, embora detenha a informação, se cala por medo.

Quanto ao médico atestante, sua conscientização a respeito da importância do preenchimento adequado da DO passa pela formação 
acadêmica e tem como entrave o receio de envolvimentos futuros com autoridades policiais ou judiciais. Visando a redução desse problema, o Ministério da Saúde em 1994 inclui no campo VI da DO (morte não natural - prováveis circunstâncias da morte) a observação "informações de caráter epidemiológico". Ao que parece, esse adendo não está obtendo o impacto esperado, conforme pode ser evidenciado pela baixa concordância entre a classificação da causa básica emitida pelo IML e a encontrada no presente estudo.

É preciso salientar, com certa preocupação, que mesmo se tendo avaliado todos os óbitos por causa externa em menores de 18 anos, nenhum pode ser codificado como sendo por Síndrome da Criança Espancada e outras formas de maus tratos. Esse fato decorreu de terse acatado as informações policiais e por não se ter realizado entrevistas domiciliares ou hospitalares, as quais certamente teriam contribuído para melhor elucidar estes eventos. No entanto, algumas informações contidas nos laudos necroscópicos insinuam a ocorrência de maus tratos. Três casos classificados como sendo acidentais ou inespecíficos (E925 e E988 em 1995 e Y34 em 1996) têm relatos sugestivos de violência como, por exemplo, fratura de osso parietal direito extensiva ao occipital por ação contundente; aparelho gessado na perna além de equimoses e hematomas na face e região lombar, hemoperitônio, hemopericárdio, etc. Ainda que este ponto certamente necessite ser melhor investigado, não deve passar em branco que, em se confirmando a ausência completa dessa categoria de óbitos, de um lado, reforçaria a pouca importância dada ainda à violência familiar em nosso meio, e de outro, serviria para nos alertar às muitas trágicas mas previníveis mortes que, possivelmente, ocorrem no Brasil.

É necessário ressaltar também que não foi identificado pelo IML nenhum óbito como sendo por causa externa em menores de um ano. Todos foram considerados como morte natural, i.e., uma mera evolução de processo mórbido. Também aqui seria necessário maio-

\section{Agradecimentos}

À Dra. Thelma Marly Abreu Mendes (Secretaria Municipal de Saúde de Duque de Caxias - SMS-DC) pela replicação na coleta de dados do Instituto Médico Legal (IML), à Marileide da Silva Dantas (SMS-DC) pela codificação da causa básica da morte e à Angela Cascão (Secretaria Estadual de Saúde do Rio de Janeiro SES-RJ) pela consultoria técnica. Michael E. Reichen- res investigações, pois o velamento de mortes intencionais neonatal e pós neonatal, se detectado, merece enfrentamento.

Os resultados apresentados corroboram estudos anteriores de que o IML, embora possua informações suficientemente detalhadas a respeito de cada morte, não as transfere aos órgãos responsáveis pela codificação via DO. Nesses, por sua vez, quando as informações da DO são usadas para complementar as do IML, ocorre uma melhora considerável, propiciando uma informação mais fidedigna sobre as mortes por causas externas.

Embora a SMS-DC, acatando diretrizes e orientações do MS e da SES-RJ, tenha encontrado uma forma de mitigar a falha oriunda do IML e melhorar consideravelmente a qualidade das informações de mortalidade por causas externas no município, é preciso questionar e elucidar, em estudos futuros, se a complementação da DO também vem sendo realizada em larga escala em outros municípios e estados. Vale ressaltar que má-classificações decorrentes do preenchimento incompleto ou incorreto da DO por legistas, podem gerar importantes distorções nas casuísticas oficiais de mortalidade por causas externas, o que, por sua vez, pode acarretar propostas e adoções de políticas públicas inadequadas. Também é importante perceber que uma busca ativa de informações na imprensa (consulta diária aos jornais) e no IML na tentativa de qualificar o óbito e complementar a DO, implica em custos financeiros e responsabilidades adicionais às atividades inerentes ao serviço.

Por fim, é importante não perder-se a perspectiva de que, mesmo se um caminho alternativo e, por sinal, efetivo, esteja sendo tentado no nível das Secretarias, a responsabilidade social e legal de informar a causa básica do óbito continua sendo do médico legista. Esforços conjuntos no âmbito das instâncias pertinentes - Ministério da Saúde, Conselho Federal de Medicina, Secretaria de Segurança Pública, etc. - devem ser empreendidos para buscar soluções permanentes e que não infrinjam as normas vigentes. heim recebeu apoio da Fundação de Amparo à Pesquisa do Estado do Rio de Janeiro - FAPERJ (proc. E26/171.223/98 e E-26/150.893/99) e Conselho Nacional de Desenvolvimento Científico e Tecnológico CNPq (proc. 300234/94-5). Pesquisa subvencionada principalmente pela SMS-DC. 


\section{Referências}

BRASIL, 1998. Código de Processo Penal (Decreto Lei no 3689/41). 38a Ed. São Paulo: Editora Saraiva.

CARVALHO, M. L. \& SILVER, L. D., 1995. Confiabilidade da declaração da causa básica de óbitos neonatais: Implicações para o estudo da mortalidade prevenível. Revista de Saúde Pública, 29:342-348.

COHEN, J., 1960. A coefficient of agreement for nominal scales. Educational and Phsychological Measurement, 20:37-46.

CDC (Centers for Disease Control and Prevention), 1990. Epi Info - User's Guide. Atlanta: CDC.

FLEISS, J. L.; COHEN, J. \& EVERITT, B. S., 1969. Large sample standard errors of kappa and weighted kappa. Psychological Bulletin, 72:323-327.

FONSECA, L. A. M. \& LAURENTI, R. A., 1974. A qualidade da certificação médica da causa de morte em São Paulo, Brasil. Revista de Saúde Pública, 8:21-29.

LADEIRA, R. M. \& GUIMARÃES, M. D. C., 1998. Análise da concordância da codificação de causa básica de óbito por acidentes de trânsito. Revista de Saúde Pública, 32:133-137.

LANDIS, J. R. \& KOCH, G. G., 1977. The measurement of observer agreement for categorical data. Biometrics, 33:159-174.

LAURENTI, R., 1997. Acidentes e violências/lesões e envenenamentos e a 10a revisão da Classificação Internacional de Doenças. Revista de Saúde Pública, 31(Sup. 4):55-58.

LAURENTI, R. \& MELLO-JORGE, M. H. P., 1983. O Atestado de Óbito. São Paulo: Centro Brasileiro de Classificação de Doenças.

LAURENTI, R. \& MELLO-JORGE, M. H. P., 1987. O Atestado de Óbito. 2a Ed. São Paulo: Centro Brasileiro de Classificação de Doenças.

MELLO-JORGE, M. H. P., 1982. Mortalidade por causas violentas no Município de São Paulo, Brasil. IV - A situação em 1980. Revista de Saúde Pública, 16:19-41.

MELLO-JORGE, M. H. P., 1988. Investigação Sobre a Mortalidade por Acidentes e Violências na Infância. Tese de Livre Docência, São Paulo: Faculdade de Saúde Pública, Universidade de São Paulo.

MELLO-JORGE, M. H. P., 1990. Situação atual das estatísticas oficiais relativas à mortalidade por causas externas. Revista de Saúde Pública, 24:217223.

MELLO-JORGE, M. H. P. \& GOTLIEB, S. L. D., 1998. As Condições de Saúde no Brasil, São Paulo. Relatório Final. São Paulo: Ministério da Saúde/Organizacão Pan-Americana de Saúde.

MINAYO, M. C. S., 1994. A violência social sob a perspectiva da saúde pública. Cadernos de Saúde Pública, 10(Sup. 1):7-18.

MONTEIRO; G. T. R.; KOIFMAN, R. J. \& KOIFMAN, S., 1997a. Confiabilidade e validade dos atestados de óbito por neoplasias. I. Confiabilidade da codificação para o conjunto das neoplasias no Estado do Rio de Janeiro. Cadernos de Saúde Pública, 13(Sup. 1):39-52.

MONTEIRO, G. T. R.; KOIFMAN, R. J. \& KOIFMAN, S. 1997b. Confiabilidade e validade dos atestados de óbito por neoplasias. II. Validação do câncer do estômago como causa básica dos atestados de óbito no Município do Rio de Janeiro. Cadernos de Saúde Pública, 13(Sup. 1):53-65.

MORIYAMA, I. M., 1989. Problems in measurement of accuracy of cause of death statistics. American Journal of Public Health, 79:1349-1350.

NJAINE, K.; SOUZA, E. R.; MINAYO, M. C. S. \& ASSIS, S. G., 1997. A produção da (des) informação sobre violência: Análise de uma prática discriminatória. Cadernos de Saúde Pública, 13:405-414.

OMS (Organização Mundial da Saúde), 1975. Manual de Classificação Estatística Internacional de Doenças, Lesões e Causas de Óbito. 9ạ Revisão, v. 1. São Paulo: Centro Brasileiro de Classificação de Doenças em Português.

OMS (Organização Mundial da Saúde), 1993. Manual de Classificação Estatística Internacional de Doenças e Problemas Relacionados à Saúde. 10a Revisão. São Paulo: Centro Brasileiro de Classificação de Doenças em Português.

OPS (Organización Panamericana de la Salud), 1994. Salud y Violencia: Plan de Acción Regional. Washington, DC: OPS. (mimeo.)

PUFFER, R. R. \& GRIFFITH, G. W., 1968. Características de la Mortalidad Urbana. Publicación Científica 151. Washington, DC: Organización Panamericana de la Salud.

REICHENHEIM, M. E. \& WERNECK, G. L., 1994. Anos potenciais de vida perdidos no Rio de Janeiro, 1990. As mortes violentas em questão. Cadernos de Saúde Pública, 10(Sup. 1):188-198.

SHROUT, P. E., 1998. Measurement reliability and agreement in psychiatry. Statistical Methods in Medical Research, 7:301-317.

SOUZA, E. R., 1991. Violência Velada e Revelada: Estudo Epidemiológico da Mortalidade por Causas Externas em Duque de Caxias, Rio de Janeiro. Dissertação de Mestrado, Rio de Janeiro: Escola Nacional de Saúde Pública, Fundação Oswaldo Cruz.

SOUZA, E. R., 1994. Homicídios no Brasil: O grande vilão da saúde pública na década de 80 . Cadernos de Saúde Pública, 10(Sup. 1):45-60.

SZWARCWALD, C. L., 1987. Mortalidade por Causas Externas nas Capitais das Grandes Regiões Metropolitanas Brasileiras, 1977-1985. São Paulo: II Congresso Brasileiro de Saúde Coletiva. (mimeo.)

ZALUAR, A.; NORONHA, J. C. \& ALBUQUERQUE, C., 1994. Violência: Pobreza ou fraqueza institucional? Cadernos de Saúde Pública, 10(Sup. 1):213217. 